\title{
Perturbative Solutions of Hamilton Jacobi Bellman Equations in Robust Decision Making
}

\author{
Fabio Trojani $^{a *} \quad$ Paolo Vanini $^{a, b}$ \\ ${ }^{a}$ Institute of Finance, University of Southern Switzerland \\ ${ }^{b}$ Zurich Cantonal Bank
}

First version: September 1999

This version: April 2002

\begin{abstract}
This paper makes use of perturbation theory to solve analytically a class of robust control problems implied by Anderson, Hansen and Sargent (2000) (AHS (2000)) model of a preference for robustness. For the constant opportunity set model, we provide (i) asymptotic expressions that characterize to any order in perturbation theory the implied value function, (ii) closed form expressions for the optimal policies up to second order and (iii) parameter constraints that ensure convergence of the whole perturbation series to a classical solution of the robust Hamilton Jacobi Bellman equation.
\end{abstract}

KeYwords: Hamilton-Jacobi Bellman Equations, Model Misspecification, Perturbation Theory, Robust Decision Making.

Jel Classification: C60, C61, G11.

\footnotetext{
${ }^{*}$ Correspondence address: Fabio Trojani, Institute of Finance, University of Southern Switzerland, Via Buffi 13, CH-6900 Lugano, e-mail: Fabio.Trojani@lu.unisi.ch. Fabio Trojani and Paolo Vanini gratefully acknowledge the financial support of the Swiss National Science Foundation (grant 12-65196.01 and NCCR FINRISK, respectively).
} 


\section{Introduction}

It is a well-known characteristic of intertemporal control problems maximizing some version of an expected utility functional to imply optimality conditions that are difficult to solve in closed form. Existence of closed form solutions depends typically on assumptions regarding the class of utility functions used, the functional form of the dynamics for the underlying state variables, existence of intermediate consumption, and further related aspects.

In his seminal work in the late sixties Robert Merton $(1969,1971)$ solved in closed form some intertemporal consumption / investment control problems maximizing the expected utility implied by an hyperbolic absolute risk aversion utility function and a geometric Brownian Motion dynamics. However, due to the complexity of the arising optimality conditions it has been clear already since Merton's work that finding analytical solutions for this class of problems would happen to be the exception rather than the rule ${ }^{1}$.

In this paper we focus on some max-min robust versions of the original Merton's problem, that have been recently proposed by Anderson, Hansen and Sargent (2000) (AHS (2000)) to model an aversion to misspecification via a preference for robustness in intertemporal optimal decision making. For this class of models no analytical solutions are known, even for the simplest model setting with Constant Relative Risk Aversion utilities of current consumption and geometric Brownian Motion dynamics. Indeed, it turns out that AHS (2000)'s model implies value functions and Hamilton Jacobi Bellman (HJB) equations that are even more difficult to solve than in the standard (non robust) case because of some intrinsic inhomogeneities. Thus, the analysis of this class of models has been so far limited to providing numerical descriptions of the implied value functions and optimal policies.

This paper makes use of perturbation theory to solve analytically the robust control problems implied by AHS (2000) model of a preference for robustness ${ }^{2}$. Specifically, we compute for the constant opportunity set case (i) asymptotic expressions that characterize to any order in perturbation theory the implied value function, (ii) closed form expressions for the solution up to second order, and (iii) parameter constraints that ensure convergence of the whole perturbation series and existence of a classical solution. Despite the non homogenous structure of the robust HJB equations, the constructive procedure adopted to write the candidate solution as a perturbation series shows that the control problems implied by AHS (2000) robustness specification have some strong intrinsic symmetries which can be in principle exploited to solve the model also for more general settings than the pure constant opportunity set case.

\footnotetext{
${ }^{1}$ Some examples of papers providing closed form solutions for some versions of the original Merton's problem are Cox and Huang (1989), Kim and Omberg (1996), Liu (1998), Cuoco (1997) and Wachter (1998).

${ }^{2}$ For a discussion on the distinction between models based on robust control theory (as in AHS (1998, 2000), Hansen et. al (2001) and Hansen, Sargent and Tallarini (1999)) and intertemporal ambiguity models extending the atemporal setting of Gilboa and Schmeidler (1989), see Chen and Epstein (2000).
} 
Recently, perturbation theory has been shown in Kogan and Uppal (2000) to be a powerful tool that naturally provides finite-order approximations for the relevant quantities in standard (non robust) Merton's models with intermediate consumption, state dependent opportunity sets and portfolio constraints. This paper considers robust versions of a Merton $(1969,1971)$ consumption/portfolio optmization problem, that are characterized by the solution of a more general max-min stochastic game. Specifically, we compute a perturbative solution for the value functions implied by these models to any order in perturbation theory. Moreover, we fully characterize convergence of the perturbation series to a classical solution of the robust optimization problem in dependence of the given model parameters. This provides a sound motivation for applying perturbative approaches in such model settings, beyond the one implied by a pure formal use of perturbation theory.

The paper is organized as follows. Section 2 defines the robust control problem implied by AHS (2000) specification of a preference for robustness. Existence of a viscosity solution is discussed in Section 3. Some first and second order perturbative solutions are presented in Section 4. In Section 5, the perturbative solution of the AHS (2000) robust control problem is computed to all orders in perturbation theory and the issue of convergence of the whole perturbation series to a solution of the robust optimization problem is considered. Section 6 summarizes and concludes.

\section{A Robust Merton's Model}

There are two assets, a risk free asset with price $B_{t}$ at time $t$ and a risky asset with price $P_{t}$ at time $t$ whose dynamics are given by

$$
\begin{aligned}
d B_{t} & =r B_{t} d t \\
d P_{t} & =\alpha P_{t} d t+\sigma P_{t} d Z_{t},\left(Q_{0}\right)
\end{aligned}
$$

for given $B_{0}, P_{0}$ and a $Q_{0}$-Brownian motion $\left(Z_{t}\right)$. The drift, volatility and riskless rate $\alpha, \sigma, r$ are constant. We consider agents with time preference parameter $\rho \geq 0$ and power utility $u(\cdot)$ of current consumption $c_{t}$ given by

$$
u(c)=\frac{c^{\gamma}-1}{\gamma} \quad, \quad \gamma<1
$$

For $\gamma \rightarrow 0$ the $\log$ utility case is obtained. Each agent allocates at each date $t$ a fraction $w_{t}$ of current individual wealth $W_{t}$ to risky assets, yielding the current wealth dynamics:

$$
d W_{t}=\left[w_{t} W_{t}(\alpha-r)+r W_{t}-c_{t}\right] d t+w_{t} W_{t} \sigma d Z_{t}
$$

To introduce model misspecifications and preferences for robustness, we follow AHS (2000). Model misspecifications are represented by absolutely continuous

measures $Q$. This defines a process $\nu$ via the Radon Nikodym derivative for any 
pair of absolutely continuous measures $Q_{0}$ and $Q$, i.e.

$$
\frac{d Q}{d Q_{0}}\left(P_{t}\right)=\nu\left(P_{t}\right), t \geq 0 .
$$

Model discrepancy between $Q$ and $Q_{0}$ is measured by the relative entropy process $I_{t}(\nu)$ defined by

$$
I_{t}(\nu)(s)=E_{Q_{0}}\left[\nu\left(P_{t}\right) \cdot \log \nu\left(P_{t}\right) \mid P_{0}=s\right] .
$$

To set up the optimization problem of a robust agent, we introduce the distorted semigroups $\left(T_{t}^{\nu}\right)$ acting on suitable test functions $\phi$ by

$$
T_{t}^{\nu}(\phi)(s):=E\left(\nu\left(P_{t}\right) \phi\left(P_{t}\right) \mid P_{0}=s\right) .
$$

The infinite horizon multiplier robust control problem in AHS (2000) and Hansen et al. (2001) is defined by

$$
J\left(W_{0}\right)=\sup _{\left(c_{s}\right),\left(w_{s}\right)} \inf _{\nu}\left[T_{t}^{\nu}\left(\int_{0}^{\infty} \exp (-\rho \tau)\left(u\left(c_{\tau}\right)+\frac{1}{\vartheta} I_{\tau}(\nu)\right) d \tau\right)\right],
$$

for some $\vartheta \geq 0$ and subject to (1), $(2)^{3}$. The HJB differential equation for the robust value function $J\left(W_{0}\right)$ reads

$$
0=\sup _{c, w}\left\{u(c)-\rho J+\inf _{\nu>0}\left(\frac{1}{\vartheta} \cdot I^{\prime}(\nu)+\mathcal{A}^{\nu} J\right)\right\},
$$

where

$$
I^{\prime}(\nu):=\lim _{t \rightarrow 0} \frac{I_{t}(\nu)}{t}
$$

and

$$
\mathcal{A}^{\nu} J:=\lim _{t \rightarrow 0} \frac{T_{t}^{\nu}(J)-J}{t}
$$

is the infinitesimal generator of the distorted semigroup $\left(T_{t}^{\nu}\right)$, evaluated at $J$.

This equation characterizes the value function of the zero-sum game between a malevolent player (selecting a worst case model $\nu^{w c}$ ) and a robust agent (choosing optimal consumption and investment rules $c_{t}, w_{t}$ ) who is rationally considering the possibility that the first agent will select a least favorable model from the set of relevant model misspecifications ${ }^{4}$.

Generally, (5) and (6) are not easily computed. However, in the diffusion setting it follows (cf. AHS (2000), p. 25) that solving (4) is equivalent to solving the single agent HJB equation

$$
\begin{aligned}
0= & \sup _{c, w}\left(\frac{c^{\gamma}-1}{\gamma}-\rho J+(w W(\alpha-r)+r W-c) \cdot J_{W}\right. \\
& \left.+\frac{1}{2} w^{2} W^{2} \sigma^{2}\left(J_{W W}-\vartheta \cdot J_{W}^{2}\right)\right) .
\end{aligned}
$$

\footnotetext{
${ }^{3}$ The relationship between this model and the so-called "constraint robust control problem" is discussed in Hansen et al. (2001).

${ }^{4}$ Equation (4) is called an Isaacs-Bellman equation in stochastic game theory but we continue to call it in the sequel "HJB equation".
} 
Thus, the optimal policies are

$$
\begin{aligned}
c & =\left(J_{W}\right)^{\frac{1}{\gamma-1}} \\
w & =-\frac{J_{W}}{\sigma^{2} W\left(J_{W W}-\vartheta \cdot J_{W}^{2}\right)} \cdot(\alpha-r) .
\end{aligned}
$$

Inserting (8), (9) in (7) finally gives the optimality condition for the robust value function

$$
\begin{aligned}
0 & =\frac{(1-\gamma)\left(J_{W}\right)^{\frac{\gamma}{\gamma-1}}-1}{\gamma}-\rho J-\frac{1}{2} \frac{(\alpha-r)^{2} J_{W}^{2}}{\sigma^{2}\left(J_{W W}-\vartheta \cdot J_{W}^{2}\right)}+r W J_{W} \\
& =: \mathbf{D}_{\gamma, \vartheta}(J(W)),
\end{aligned}
$$

with $\mathbf{D}_{\gamma, \vartheta}$ the differential operator parameterized by the robustness parameter $\vartheta$ and the risk aversion index $\gamma$. The boundary condition for this differential equation is

$$
\lim _{\gamma \rightarrow 0} J(W)=J_{\log }(W),
$$

where $J_{\log }(W)$ is the known value function of a robust, log-utility investor. The goal of the paper is to solve perturbatively (10) subject to the boundary condition (11). This defines a robust intertemporal control problem of an agent with risk aversion index $\gamma<1$ and robustness parameter $\vartheta \geq 0$.

\section{Existence of a Viscosity Solution}

A sound mathematical foundation for the existence of a viscosity solution to the two-player game (4) requires results in Fleming and Souganidis (1989), Souganidis (1999, for the existence of the value function), Fleming and Soner (1993, for growth conditions in infinite time horizon problems) and Yong and Zhou (1999, for parametric problems). Before analyzing the HJB equation (7) using perturbation theory, we thus first discuss some basic issues related to the existence of a viscosity solution. We start by considering the wealth dynamics

$$
d W_{t}=\underbrace{\left[w_{t} W_{t}(\alpha-r)+r W_{t}-c_{t}\right]}_{=: f(W, w, c)} d t+\underbrace{w_{t} W_{t} \sigma}_{=: g(W, w)} d Z_{t} .
$$

A first condition to ensure existence of a viscosity solution is the existence of a constant $C>0$ such that the bounds

$$
\left|f_{W}\right| \leq C,\left|g_{W}\right| \leq C,
$$

for the derivatives $f_{W}, g_{W}$, hold and such that the Lipschitz conditions

$$
|f| \leq C(1+|W|+||(w, c)||)),|g| \leq C(1+|W|+||(w, c)||)
$$


hold, denoting by $\|\cdot\|$ the Euclidean norm. If $|w| \leq M$, i.e. short-selling and borrowing are restricted, setting

$$
C=\max \left\{M|\alpha-r|+r,(M+1) r, \frac{M|\alpha-r|}{r(M+1)}, \frac{1}{r(M+1)}\right\}
$$

implies (13). The next step is to ensure continuity of the utility index $L(t, c)=$ $e^{-\rho t} u(c)$ and to satisfy the growth condition

$$
|L(t, c)| \leq C_{1}\left(1+|c|^{k}\right), k \text { a constant } .
$$

If $c>0$ and $C_{1}=\gamma$, this growth condition is satisfied and the existence of a viscosity solutions follows (see for example Theorem 5.4 in Souganidis (1999)). Finally, we have to consider behavior of a solution to (10) in the limit $\gamma \rightarrow 0$, in order to satisfy the boundary condition (11). Thus, further conditions need to be satisfied. First, $L, f, g$ have to be uniformly continuous in the state variable $W$, the decision variables $w, c$, and the parameter $\gamma$. Second, the Lipschitz conditions

$$
|\phi(t, W, c, w)-\phi(t, \hat{W}, c, w)| \leq C_{2}|W-\hat{W}|, \phi(t, 0, w, c) \mid \leq C_{3}, \forall t, W, \hat{W}
$$

have to hold for $\phi=L, f, g$. Finally, the limits

$$
\lim _{\gamma \rightarrow 0}\left|\phi_{\gamma}(t, W, c, w)-\phi_{\gamma=0}(t, W, c, w)\right|=0
$$

have to hold uniformly in $t, w, c$ and uniformly in $W$ on compact sets.

Given the above bounds for $w, c$, uniform and Lipschitz continuity of $L, f, g$, in $W, c, w$ follow. A bit more involved is the uniform continuity of $L$ w.r.t. to the parameter $\gamma$. After some calculations, we get

$$
\left|L\left(\gamma_{1}\right)-L\left(\gamma_{2}\right)\right| \leq C_{4}\left|\gamma_{1}-\gamma_{2}\right|,
$$

with $C_{4}=\max \left\{\frac{1}{2} \log (c)^{2}, c(\log (c)-1)+1\right\}$. Therefore, uniform continuity follows if $c$ is bounded above and $c>0$. Summarizing, we thus have that for $w$ and $c$ bounded and for the constants $C_{i}$ defined above, a unique viscosity solution to problem (10) subject to the boundary condition (11) exists.

\section{Perturbative Solutions}

Exact analytical solutions of (10) for the limit cases $\vartheta=0$ or $\gamma=0$ are wellknown (cf. Merton (1969, 1971) and Schroder and Skiadas (1999)). They are given below for completeness, since they imply boundary conditions on the candidate solution for the general case $\vartheta>0, \gamma<1$.

- Standard Merton's Model ( $\vartheta \rightarrow 0$ ). The HJB equation is

$$
0=\frac{(1-\gamma)\left(J_{W}\right)^{\frac{\gamma}{\gamma-1}}-1}{\gamma}-\rho J-\frac{q^{2} J_{W}^{2}}{J_{W W}}+r W J_{W}=: \mathbf{D}_{\gamma, 0}(J(W)),
$$


where

$$
q=\frac{(\alpha-r)^{2}}{2 \sigma^{2}}
$$

and with solution

$$
\begin{aligned}
J^{(0)}(W) & =\frac{1}{\rho} \frac{\left(e^{g_{\gamma, 0}} W\right)^{\gamma}-1}{\gamma}, \\
g_{\gamma, 0} & =\ln (\rho)+\frac{(\gamma-1)}{\gamma} \ln \left(\frac{\gamma}{1-\gamma}\left(\frac{1}{\gamma}+\frac{q}{(\gamma-1) \rho}-\frac{r}{\rho}\right)\right) .
\end{aligned}
$$

- Robust log utility investor $(\gamma \rightarrow 0)$. The HJB equation is

$$
0=-\ln \left(J_{W}\right)-1-\rho J-\frac{q J_{W}^{2}}{J_{W W}-\vartheta \cdot J_{W}^{2}}+r W J_{W}=: \mathbf{D}_{0, \vartheta}(J(W))
$$

with solution

$$
\begin{aligned}
J_{\log }(W) & =\frac{1}{\rho}\left(\ln (W)+g_{0, \vartheta}\right) \\
g_{0, \vartheta} & =\ln (\rho)-1+\frac{q}{\rho\left(1+\frac{\vartheta}{\rho}\right)}+\frac{r}{\rho} .
\end{aligned}
$$

To provide a solution to (10) we adopt a power series approach of the form

$$
J(W)=\sum_{i=0}^{\infty} \frac{\vartheta^{i}}{i !} J^{(i)}(W) \quad, \quad W \geq 0
$$

The functions $\left(J^{(i)}\right)_{i \in \mathbf{N}}$ are determined recursively, starting from the zeroth order term $J^{(0)}$ which is the standard Merton's solution (15). Inserting formally this power series in the HJB equation (10) and ordering the result in powers of the coefficients $\vartheta$, we get a an infinite hierarchy $i=1,2, \ldots$, of differential equations for the function $J^{(i)}, i=1,2, \ldots$. We proceed as follows:

- We first derive the hierarchy of equations. It turns out that for each $i$ an inhomogeneous Euler equation is obtained, whose homogeneous part is independent of the stage $i$ of the hierarchy. Dependence on $i$ shows up in the (complicated) inhomogeneous part.

- For each Euler equation appropriate boundary conditions are defined.

- We solve the hierarchy explicitly for $i=1,2$. These solutions provide us with the first and second order robust corrections to the solution in the standard Merton model and with exact comparative statics for the implied robust optimal policies.

- Finally, we discuss convergence of the whole perturbation series and construct the value function approximation of the robust Hamilton Jacobi Bellman equation to any order. 
We introduce the following notations which are used in Theorem 1 below. The inhomogeneity of order $k$ is defined by

$$
R^{(k)}:=\mathbf{D}_{\gamma, \vartheta}\left(S^{(k)}(W)\right) \quad, \quad k \geq 0
$$

with the partial sums

$$
S^{(k)}=\sum_{i=0}^{k} \frac{\vartheta^{i}}{i !} J^{(i)} \quad, \quad k \geq 0
$$

Finally, we define for any order $k \geq 0$ the boundary condition

$$
\lim _{\gamma \rightarrow 0} S^{(k)}(W)=J_{\log }^{(k)}(W)
$$

where $J_{l o g}^{(k)}$ is the expansion of the solution (16) up to order $k$ in the robustness parameter $\vartheta$. These boundary conditions ensure that each order perturbation theory converges to the corresponding asymptotic solution of a robust log-utility investor. The next theorem characterizes the desired solutions and gives analytical expressions for the first order term.

Theorem 1 Assume that the differential equation (10) with the boundary condition (11) has a classical solution ${ }^{5} J(W)$.

1. For any $k \geq 1$ the function $J^{(k)}$ in the power series (17) solves the inhomogeneous Euler differential equation

$$
0=-\frac{R^{(k-1)} k !}{\vartheta^{k}}+\rho J^{(k)}+a_{1} W J_{W}^{(k)}-a_{2} W^{2} J_{W W}^{(k)}
$$

with

$$
a_{1}=\left(\frac{e^{\gamma g_{\gamma}, 0}}{\rho}\right)^{\frac{1}{\gamma-1}}-r+\frac{2 q}{(\gamma-1)}, a_{2}=\frac{q}{(\gamma-1)^{2}} .
$$

2. For $k=1$ the Euler differential equation (19) reads

$$
0=-a_{2}\left(\frac{\left(e^{g_{\gamma, 0}} W\right)^{\gamma}}{\rho}\right)^{2}+\rho J^{(1)}+a_{1} W J_{W}^{(1)}-a_{2} W^{2} J_{W W}^{(1)}
$$

3. The solution $J^{(1)}$ to the Euler differential equation (21) such that the boundary condition (18) is satisfied for $S^{(1)}$ is

$$
J^{(1)}(W)=\frac{\left(e^{2 g_{\gamma, 0}} W^{2}\right)^{\gamma}}{C_{1}},
$$

\footnotetext{
${ }^{5}$ I.e. the solution is an at least twice continuously differentiable function of wealth.
} 
where

$$
C_{1}=\rho^{2}\left(2 \gamma\left(2 \gamma+\delta_{1}-1\right)+\delta_{2}\right), \delta_{1}=-\frac{a_{1}}{a_{2}}, \delta_{2}=-\frac{\rho}{a_{2}} .
$$

Hence, the solution to the robust HJB equation (10) up to $O\left(\vartheta^{2}\right)$ is

$$
J=J^{(0)}+\vartheta J^{(1)}+O\left(\vartheta^{2}\right)
$$

The second order term in the approximation $S^{(2)}$ is more complicated than the first order one. However, the proof of Theorem 1 already describes how it can be computed. The result is the next corollary.

Corollary 2 The solution to the robust HJB equation (10) up to $O\left(\vartheta^{3}\right)$ is

$$
J=J^{(0)}+\vartheta J^{(1)}+\frac{\vartheta^{2}}{2} J^{(2)}+O\left(\vartheta^{3}\right)
$$

where the zeroth and first order terms are given in (14) and Theorem 1, respectively, and

$$
J^{(2)}=\frac{-q_{3} W^{3 \gamma}}{q_{1}+3 \gamma\left(q_{2}+(3 \gamma-1) q_{4}\right)}+C_{2} W^{\frac{1}{2}+\frac{q_{2}+q_{5}}{2 q_{4}}},
$$

wit the constants $q_{i}$ given in the footnote below ${ }^{6}$.

The second order term $J^{(2)}$ in the perturbation series is obtained by solving (19) for $k=2$ using the variation of constant formula and the explicit expression for $R^{(1)}$ in Theorem 1 . Notice that the constant $C_{2}$ is calculated using the boundary condition (18) for $k=2$. For the sake of brevity we omit the lengthy calculations in the proof of Corollary 2.

\section{$5 \quad$ First Order Asymptotics for Robust Optimal Policies}

Inserting the first order asymptotics (22) for the robust value function solution in (8), (9), the desired first order approximations of the robust optimum consumption and investment policies are obtained ${ }^{7}$.

$$
\begin{aligned}
q_{1} & =-\frac{1}{2} \rho, K_{0}=\frac{e^{\gamma g_{\gamma, 0}}}{\rho \gamma}, K_{1}=\frac{e^{\gamma g_{\gamma, 0}}}{C_{1}} \\
q_{2} & =\frac{1-\gamma\left(K_{0} \gamma\right)^{\frac{\gamma}{\gamma-1}}+K_{0} \gamma(-2 q+(\gamma-1) r)}{2 K_{0}(\gamma-1) \gamma} \\
q_{3} & =\frac{-4\left(\frac{1}{2} K_{1}^{2}(\gamma-1)^{2}\left(K_{0} \gamma\right)^{\frac{\gamma}{\gamma-1}}-K_{0}^{3} K_{1} \gamma^{2} q+\frac{1}{4} K_{0}^{5} \gamma^{3} q+K_{0} K_{1}^{2} \gamma^{3} q\right)}{K_{0}^{2}(\gamma-1)^{3}} \\
q_{4} & =\frac{q}{2(\gamma-1)^{2} \gamma}, q_{5}=\sqrt{\left(q_{2}-q_{4}\right)^{2}-4 q_{1} q_{4}}
\end{aligned}
$$

$C_{1}, g_{\gamma, 0}$ and $q$ are defined in Theorem 1 and in (14), respectively.

${ }^{7}$ Using Theorem 1, Corollary 2 and equations (8), (9), second order optimal policies can be also easily derived. 
Proposition 3 The first order asymptotics to the exact optimal policies (8), (9), are:

1. For consumption,

$$
c=\left(\frac{e^{\gamma g_{\gamma, 0}}}{\rho}\right)^{\frac{1}{\gamma-1}} W\left(1-\frac{\vartheta \rho}{(1-\gamma) C_{1}}\left(e^{g_{\gamma, 0}} W\right)^{\gamma}\right)+O\left(\vartheta^{2}\right) .
$$

2. For the risky asset allocation,

$$
w=\frac{\alpha-r}{\sigma^{2}(1-\gamma)}\left(1-\vartheta \cdot \frac{C_{1}-2 \gamma^{2} \rho^{2}}{\rho(1-\gamma) C_{1}}\left(e^{g_{\gamma, 0}} W\right)^{\gamma}\right)+O\left(\vartheta^{2}\right) .
$$

First order robust optimal consumption and risky allocation consist of the optimal policy of a standard Merton investor and a further precautionary component deriving because of a preference for robustness

$$
\begin{aligned}
& c=\underbrace{\left(\frac{e^{\gamma g_{\gamma, 0}}}{\rho}\right)^{\frac{1}{\gamma-1}} W}_{\text {Merton Policy }}-\underbrace{\frac{\vartheta \rho}{(1-\gamma) C_{1}}\left(e^{g_{\gamma, 0}} W\right)^{\gamma}\left(\frac{e^{\gamma g_{\gamma, 0}}}{\rho}\right)^{\frac{1}{\gamma-1}} W}_{\text {Robustness Induced Consumption Term }}+O\left(\vartheta^{2}\right), \\
& w=\underbrace{\frac{(\alpha-r)}{\sigma^{2}(1-\gamma)}}_{\text {Merton Policy }}-\underbrace{\frac{\vartheta \rho}{(1-\gamma) C_{1}} \cdot\left(e^{g_{\gamma, 0}} W\right)^{\gamma}\left(\frac{C_{1}}{\rho}-2 \gamma^{2} \rho\right) \frac{(\alpha-r)}{\sigma^{2}(1-\gamma)}}_{\text {Robustness Induced Risky Allocation Term }}+O\left(\vartheta^{2}\right) .
\end{aligned}
$$

By construction, for $\vartheta \rightarrow 0$ the robust rules converge to the standard optimal consumption and investment policies of the classical Merton $(1969,1971)$ model

$$
c \rightarrow\left(\frac{e^{\gamma g_{\gamma, 0}}}{\rho}\right)^{\frac{1}{\gamma-1}} W,(\vartheta \rightarrow 0), \quad w \rightarrow \frac{(\alpha-r)}{\sigma^{2}(1-\gamma)},(\vartheta \rightarrow 0) .
$$

On the other hand, for $\gamma \rightarrow 0$ the robust optimal policies converge to the first order asymptotics for the optimal rules of a robust logarithmic investor which are implied by the explicit solution (16):

$$
c \rightarrow \rho W+O\left(\vartheta^{2}\right),(\gamma \rightarrow 0) \quad, \quad w \rightarrow \frac{(\alpha-r)}{\sigma^{2}}\left(1-\frac{\vartheta}{\rho}\right)+O\left(\vartheta^{2}\right),(\gamma \rightarrow 0)
$$

Since for robust $\log$ utility investors optimal consumption is independent of $\vartheta$, the first order consumption approximation in (25) coincides with the exact optimal policy.

The robustness parameter $\vartheta$ affects directly both optimum consumption and allocations to risky assets. Moreover, the impact of a preference for robustness on the optimal rules is dependent on current wealth and risk aversion in a non linear way. The exact comparative statics for the optimum consumption and 
the optimal risky allocation policies are

$$
\begin{aligned}
\frac{\partial c}{\partial \vartheta} & =-\frac{\rho}{(1-\gamma) C_{1}}\left(e^{g_{\gamma, 0}} W\right)^{\gamma}\left(\frac{e^{\gamma g_{\gamma, 0}}}{\rho}\right)^{\frac{1}{\gamma-1}} W \quad, \quad \gamma<1 \quad \\
\frac{\partial w}{\partial \vartheta} & =-\frac{\rho}{(1-\gamma) C_{1}} \cdot\left(e^{g_{\gamma, 0}} W\right)^{\gamma}\left(\frac{C_{1}}{\rho}-2 \gamma^{2} \rho\right) \frac{(\alpha-r)}{\sigma^{2}(1-\gamma)} \quad, \quad \gamma<1 .
\end{aligned}
$$

For given model parameters, the direction of the impact of robustness on optimal consumption and investment is fully determined by the sign of the constants $C_{1}$ and $\frac{C_{1}-2 \gamma^{2} \rho^{2}}{C_{1}}$, which - computed explicitly - give

$$
C_{1}=\frac{2 \sigma^{2}}{(\alpha-r)^{2}} \rho^{2}\left(\left(\frac{(\alpha-r)^{2}}{\sigma^{2}}+\rho-2 r\right) \gamma^{2}+\left(\frac{(\alpha-r)^{2}}{\sigma^{2}}+2 r\right) \gamma-\rho\right),
$$

and a similar expression for $\frac{C_{1}-2 \gamma^{2} \rho^{2}}{C_{1}}$. Therefore, the roots in $\gamma$ of the second order polynomial equations $C_{1}=0$ and $C_{1}-2 \gamma^{2} \rho^{2}=0$ characterize completely the direction of the marginal impact of a preference for robustness as a function of relative risk aversion.

Figures 1 and $2^{8}$ illustrate the difference between the first order robust policies and the classical Merton policies in dependence of $W$ and $\gamma$. For the given parameter choice, we observe that the robust agent consumes more and invests less in the risky asset than a comparable Merton's investor. Moreover, as expected optimal robust consumption and risky investment are nonlinear functions of current wealth $W$. Finally, notice that although the surfaces are smooth for the plotted domain of $\gamma$, singularities will arise on a broader parameter domain. Such singularity issues will be discussed more precisely in the next sections.

\section{First and Second Order Solutions: Some Nu- merical Calculations}

We illustrate the features of the above first and second order value function approximations with some graphs based on the specific parameter choice used in Figures 1 and 2. Figure 3 plots the value functions approximations for $\gamma=0.1$ and $\vartheta=0.1$. As expected, the zeroth-order solution is an upper bound for the first and second order approximations. Moreover, for the given parameterization the difference between the first and the second order terms is much smaller than the one between the zeroth and the first order ones. This suggests that for the given parameter choice the power series convergence is fast.

Figure 4 shows the joint dependence of the second order value function approximation on current wealth $W$ and the risk aversion parameter $\gamma$. We remark, that the value function has for given wealth levels singularities w.r.t. $\gamma$ which correspond to the roots of the equation $C_{1}=0$. Therefore, the original robust

\footnotetext{
${ }^{8}$ The following parameters are chosen. The risk free rate $r=0.03$, the volatility $\sigma=0.2$, the time preference rate $\rho=0.04$ and the expected return $\alpha=0.1$.
} 


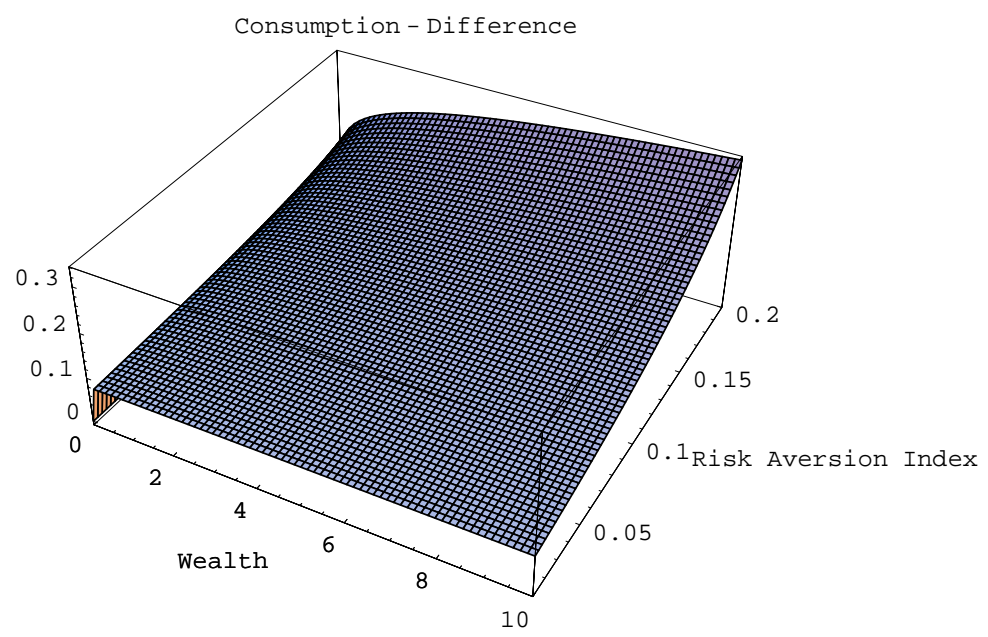

Figure 1: Difference between robust optimal consumption and the one of a standard Merton investor.

optimization problem does not have a solution for all possible parameter choices in the model. This indicates that a classical solution for the differential equation (10) can only exist on a restricted parameter set. Indeed, in the next section where we prove convergence of the perturbation series bounds on the parameter values are needed to ensure convergence. Therefore, a viscosity solution of the HJB (10) is not generally a classical solution on the whole parameter domain.

\section{Perturbative Solutions to all Orders}

In this section, we construct for any order $k \geq 1$ a solution of the HJB equation (10) with boundary condition (11), by providing conditions for the convergence of the perturbative series (17) to a smooth limit. The first step towards this goal considers a $O\left(\vartheta^{k+2}\right)$ asymptotics for the inhomogeneity $R^{(k)}(\vartheta)$.

Lemma 4 For $k \geq 1$ the inhomogeneity of order $k$ is given by

$$
R^{(k)}=-\frac{\vartheta^{k+1}}{k !} \mathbf{M}\left(J^{(k)}\right)+O\left(\vartheta^{k+2}\right),
$$

where the linear differential operator $\mathbf{M}$ is defined by

$$
\mathbf{M}\left(J^{(k)}\right)=M_{0} W^{\gamma+1} J_{W}^{(k)}-\left(M_{1} W^{\gamma+2}-M_{2} W^{-(\gamma-4)}\right) J_{W W}^{(k)}
$$




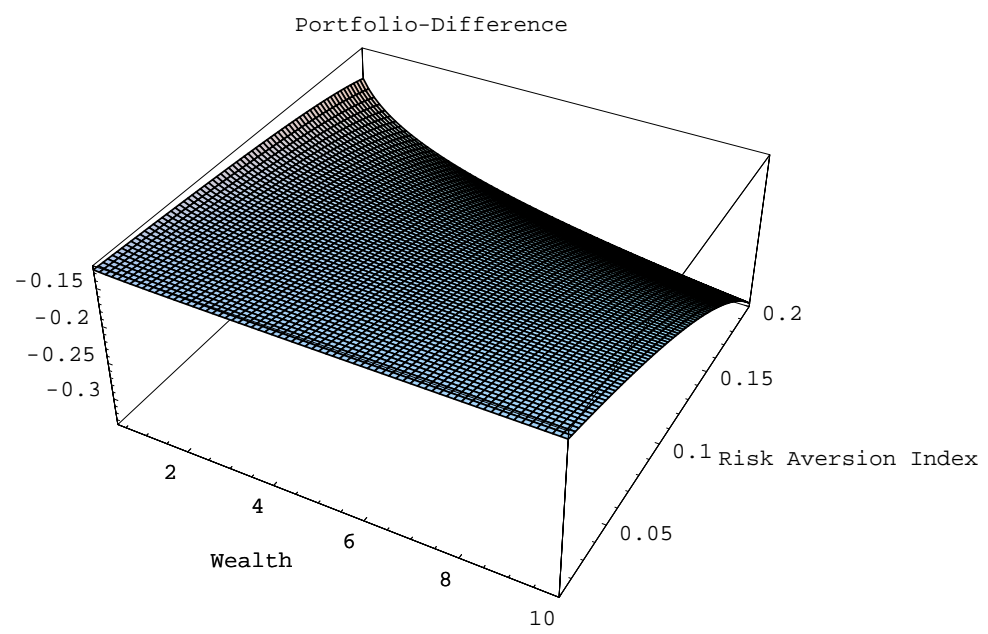

Figure 2: Difference between robust optimal investment and the one of a standard Merton investor.

with constants $M_{0}, M_{1}, M_{2}$, given by

$$
\begin{aligned}
& M_{0}=\frac{e^{\gamma g_{\gamma, 0}}}{(\gamma-1) C_{1}}\left(2 \gamma \rho\left(\frac{1}{\rho} e^{\gamma g_{\gamma, 0}}\right)^{\frac{\gamma}{\gamma-1}}+q\left(\frac{3 C_{1}-4 \gamma^{2} \rho^{2}}{\rho(\gamma-1)}\right)\right), \\
& M_{1}=\frac{2 \rho e^{\gamma g_{\gamma, 0}} q}{(\gamma-1)^{2} C_{1}}, \\
& M_{2}=\left(\frac{\rho}{\gamma-1}\right)^{3} \frac{2 q}{e^{\gamma g_{\gamma, 0}}}\left(\frac{2 \gamma(2 \gamma-1)}{C_{1}}-\frac{1}{\rho^{2}}\right) .
\end{aligned}
$$

It turns out that the inhomogeneity of order $k$ can be written, up to errors of order $O\left(\vartheta^{k+2}\right)$, as a linear combination of the first two derivatives of $J^{(k)}$. We exploit this fact in the sequel together with the hierarchy of Euler equations for $J^{(k)}, k \geq 1$, given in Theorem 1 . To clarify the structure of the argument let us recall the homogeneous differential operator (cf. also (19))

$$
\mathcal{D}=\rho+a_{1} W \partial_{W}-a_{2} W^{2} \partial_{W W}^{2}
$$

where $a_{1}, a_{2}$ have been defined in Theorem 1 . From the same Theorem and Lemma 4 the hierarchy of equations implied by (19) reads

$$
\mathcal{D} J^{(k)}=-\frac{k !}{\vartheta^{k}} R^{(k-1)}=-\mathbf{M}\left(J^{(k-1)}\right)+O(\vartheta), k \geq 1 .
$$




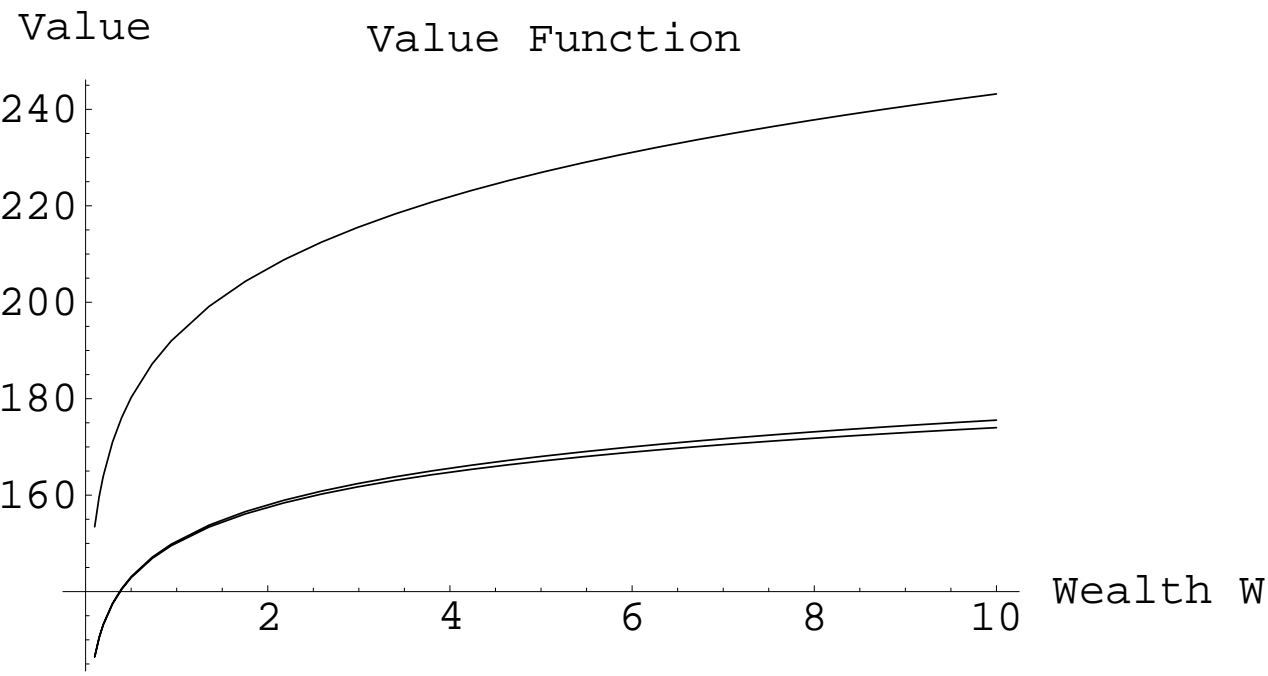

Figure 3: The value function $J^{(0)}$ for the zeroth order approximation (upper curve) in $\vartheta$, the first order one $J(W)=J^{(0)}(W)+\vartheta J^{(1)}(W)$ (lower curve) and the second order one $J(W)=J^{(0)}(W)+\vartheta J^{(1)}(W)+\frac{1}{2} \vartheta^{2} J^{(2)}(W)$ (middle curve) $(\vartheta=0.1)$.

Since the homogeneous equation $\mathcal{D} J^{(k)}=0$ has a known, $k$-independent, fundamental system, we can solve (27) using recursively $k-1$-times the variation of constant formula. For any $k \geq 1$ this determines the $k$-th order value function approximation term $J^{(k)}$ as a functional of the known zeroth order value function term $J^{(0)}$. The resulting functionals have then to be summed over $k$ to analyze the convergence of the perturbation series to a (smooth) limit.

In order to write $J^{(k)}$ as a corresponding functional of $J^{(0)}$ we first denote the fundamental system of the homogenous equation $\mathcal{D} J^{(k)}=0$ by $\left(f_{1}, f_{2}\right)=$ $\left(W^{a}, W^{b}\right)$, where

$$
a=\frac{1-\delta_{1}-\sqrt{\left(\delta_{1}-1\right)^{2}-4 \delta_{2}}}{2}, b=\frac{1-\delta_{1}+\sqrt{\left(\delta_{1}-1\right)^{2}-4 \delta_{2}}}{2},
$$

and with $\delta_{1}, \delta_{2}$ given in Theorem 1 . Further, we adopt an adequate summation notation to control for the number of arising terms when we solve recursively $J^{(k)}$ in terms of $J^{(0)}$ with the variation of constant method. Specifically, for $A=\{a, b\}$ we define the sum $\sum_{\left(a_{k}, b_{k}\right) \in A} g\left(a_{k}, b_{k}\right)$ by

$$
\sum_{\left(a_{k}, b_{k}\right) \in A} g\left(a_{k}, b_{k}\right)=g(a, b)+g(b, a) .
$$




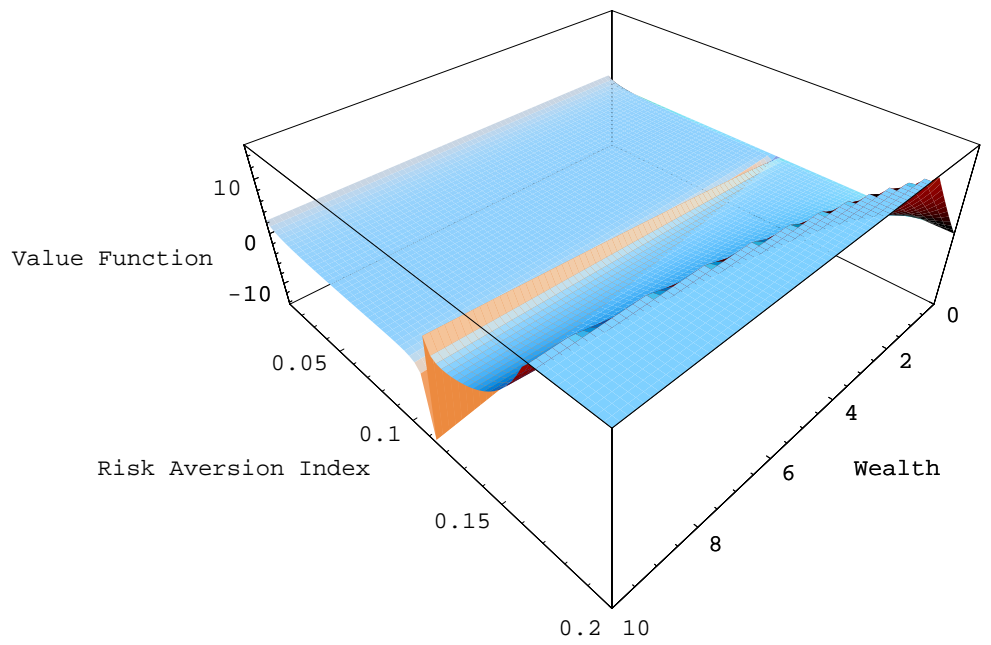

Figure 4: Plot of the second order value function as a function of wealth $W$ and the risk aversion index $\gamma(\vartheta=0.1)$.

By applying the variation of constant method to (27), while using Lemma 4 and the relevant boundary conditions in each order $k \geq 1$ of the recursion, we finally can write

$$
\begin{aligned}
J^{(k)}= & (-1)^{k} \sum_{\left(a_{k}, b_{k}\right) \in A} \sum_{\left(a_{k-1}, b_{k-1}\right) \in A} \ldots \sum_{\left(a_{1}, b_{1}\right) \in A} \\
\times & W^{a_{k}} \int W^{b_{k}} \mathbf{M}\left(W^{a_{k-1}} \int W^{b_{k-1}} \mathbf{M}(\right. \\
& \left.\left.\ldots \mathbf{M}\left(W^{a_{1}} \int W^{b_{1}} \mathbf{M}\left(J^{(0)}\right) d W\right) \ldots\right) d W\right) d W .
\end{aligned}
$$

The functional dependence of $J^{(k)}$ on $J^{(0)}$ in (28) is too complicated to be used for a direct convergence analysis of the given perturbation series. The next Lemma provides a more manageable expression where $J^{(k)}$ is written as a linear combination of powers of $W$. 
Lemma 5 The $k$-th order value function reads

$$
\begin{aligned}
J^{(k)} & =(-1)^{k} e^{\gamma g_{\gamma, 0}} \sum_{\left(a_{k}, b_{k}\right) \in A} \sum_{\left(a_{k-1}, b_{k-1}\right) \in A} \ldots \sum_{\left(a_{1}, b_{1}\right) \in A} \sum_{i_{k}=1}^{2} \sum_{i_{k-1}=1}^{2} \ldots \sum_{i_{1}=1}^{2} \\
& \times \frac{e_{i_{1}} W^{h_{i_{1}}^{0}+\sum_{n=0}^{k}\left(a_{n}+b_{n}\right)+\sum_{n=0}^{k-1} m_{i_{n}}+k-1}}{\prod_{m=0}^{k}\left(h_{i_{1}}^{0}+\sum_{n=0}^{m-1} b_{n}+\sum_{n=0}^{m-1} m_{i_{n}}+m\right)}
\end{aligned}
$$

where

$$
\begin{aligned}
m_{i_{n}} & = \begin{cases}\gamma & \text { for } i_{n}=1 \\
\gamma-6 & \text { for } i_{n}=2\end{cases} \\
e_{i_{1}} & = \begin{cases}\gamma\left(M_{0}-M_{1}(\gamma-1)\right) & \text { for } i_{1}=1, \\
\gamma M_{2}(\gamma-1) & \text { for } i_{1}=2 .\end{cases} \\
h_{i_{1}}^{0} & =\left\{\begin{array}{ll}
2 \gamma & \text { for } i_{1}=1 \\
2 \gamma-6 & \text { for } i_{1}=2
\end{array} .\right.
\end{aligned}
$$

Lemma 5 immediately implies a set of characterizing conditions for the existence of a twice continuously differentiable $k$-th order approximation term $J^{(k)}$.

Corollary 6 The $k$-th order value function approximation $J^{(k)}$ is twice continuously differentiable in $W \in(0, \infty)$ for any $k \geq 1$ if and only if

$$
\frac{e_{i_{1}}}{\prod_{m=0}^{k}\left(h_{i_{1}}^{0}+\sum_{n=0}^{m-1} b_{n}+\sum_{n=0}^{m-1} m_{i_{n}}+m\right)} \neq 0
$$

and

$$
a_{1}>0, \frac{a_{1}}{a_{2}}>2(3-\gamma),
$$

where the constants $a_{1}, a_{2}$ are given in Theorem 1.

Condition (30) in Corollary 6 guarantees that $J^{(k)}$ has no singularities due to model parameters. The second condition in (31) is technical, while the first one can be interpreted economically. Indeed, the requirement $a_{1}>0$ is equivalent to the condition

$$
\frac{\alpha-r}{\sigma^{2}(1-\gamma)}<\frac{\frac{e^{\gamma g_{\gamma, 0}}}{\rho}-r}{\alpha-r} .
$$

Therefore, the admissible set of parameters must provide an optimal investment strategy $\frac{\alpha-r}{\sigma^{2}(1-\gamma)}$ in a standard (non robust) Merton's model that is bounded by the ratio of

$$
\frac{e^{\gamma g_{\gamma, 0}}}{\rho}-r=\left(\frac{c^{*}}{W}\right)^{\gamma-1}-r
$$


i.e., the difference between the marginal utility of the optimal consumption to wealth ratio $c^{*} / W$ in the standard Merton's model and the interest rate, and the equity premium. We note that if we exclude the value $W=0$, the value function is infinitely differentiable in all wealth levels.

Lemma 5 also implies that to any order $k \geq 1$ the $k$-th order value function term $J^{(k)}(W, \gamma)$ is generically ${ }^{9}$ a continuous differentiable function on ${ }^{10}$ $(0, \infty) \times(-\infty, 1)$. Figure 4 illustrates the singularities arising in a a second order approximation of the robust value function.

We can now finally analyze the existence of a power series representation of the form

$$
J(W)=\sum_{k=0}^{\infty} \frac{\vartheta^{k}}{k !} J^{(k)}(W)
$$

for the value function implied by the robust HJB equation (10). To this end, we introduce the sequence of sets $\mathcal{S}^{(k)}, k \geq 1$, of $k$-th order singularities defined by

$$
\mathcal{S}^{(k)}=\left\{(\gamma, \rho, \alpha, \sigma, r) \in \mathbf{R}^{5} \mid \prod_{m=0}^{k}\left(2 \gamma-6+\sum_{n=0}^{m-1} b_{n}+m(\gamma-6)+m\right)=0\right\}
$$

Thus

$$
\mathbf{S}:=\bigcup_{k=1}^{\infty} \mathcal{S}^{(k)}
$$

characterizes the set of model parameter values where at least a single value function term $J^{(k)}$ has a singularity. On the complement set $\mathbf{S}^{c}$, no singularities due to the model parameters in any order perturbation theory occur.

Further, notice that the proof of Lemma 5 implies that the second condition in Corollary 6 - which gives the twice continuous differentiability of the $k$-th order value function $J^{(k)}$ - can be weakened to

$$
a_{1}>0, \frac{a_{1}}{a_{2}}>\frac{1}{k+1}(9-2 \gamma)-1-\frac{k}{k+1}(\gamma-5) .
$$

\footnotetext{
${ }^{9}$ I.e. the set of parameter choices that leads to a discontinuous value function term in $\gamma$ and $W$ has measure zero.

${ }^{10}$ This follows immediately from the denominator in (29), which determines the possible singularities of $J^{(k)}$. Indeed, when writing this term more explicitly we see that for any $k \geq 1$ the solution of the equation

$$
\prod_{m=0}^{k}\left(2 \gamma-6+\sum_{n=0}^{m-1} b_{n}+m(\gamma-6)+m\right)=0,
$$

defines the corresponding singularities set. The zero locus of (34) is a subspace of $\mathbf{R}^{5}$ spanned by the parameters $\gamma, \rho, \alpha, \sigma, r$, and is a non-generic set.
} 
This motivates introducing a set $\mathcal{C}^{(k)}$ defined by

$$
\begin{aligned}
\mathcal{C}^{(k):=} & \left\{(\gamma, \rho, \alpha, \sigma, r) \in \mathbf{R}^{5} \mid a_{1}>0\right. \\
& \left.\frac{a_{1}}{a_{2}}>\frac{1}{k+1}(9-2 \gamma)-1-\frac{k}{k+1}(\gamma-5) \text { hold true }\right\} \bigcap\left(\mathcal{S}^{(k)}\right)^{c},
\end{aligned}
$$

which identifies the parameter values for which the value function approximation $S^{(k)}$ is twice continuously differentiable. The set

$$
\mathbf{C}:=\bigcap_{k=1}^{\infty} \mathcal{C}^{(k)}
$$

then characterizes the subset of parameter values for which any $k$-th order approximation term $J^{(k)}, k \geq 1$, is twice continuously differentiable. The next Theorem gives the conditions for the convergence of the perturbation series (35) to a classical solution of the HJB equation (10) with the boundary condition (11).

Theorem 7 Consider the robust control problem (10) with the boundary condition (11) and the formal perturbation series

$$
J(W)=\sum_{k=0}^{\infty} \frac{\vartheta^{k}}{k !} J^{(k)}(W)
$$

1. If $(\gamma, \rho, \alpha, \sigma, r) \in \mathbf{C}$, then the formal perturbation series $J(W)$ exists and is twice continuously differentiable.

2. If the HJB equation (10) with the boundary condition (11) has a unique twice continuously differentiable solution for a parameter domain $\mathbf{D}$ such that $\mathbf{D} \cap \mathbf{C} \neq \emptyset$, then the formal power series $J(W)$ coincides with this solution on the parameter set $\mathbf{D} \cap \mathbf{C}$.

\section{Conclusions}

We used perturbation theory to solve the robust control problem implied by AHS (2000) definition of a preference for robustness up to all orders in perturbation theory. We derived in closed form the first and second order approximation to the robust Merton's solution. We then computed comparative statics for the optimal policies and characterized the sign of the marginal impact of a preference for robustness in terms of the model parameters. Further, we specified conditions on the model parameters which guarantee the existence of a smooth value function approximation to any order in perturbation theory. Finally, we characterized the convergence of the formal perturbation series implied by the obtained finite order approximations to a solution of the robust HJB equation. 


\section{Appendix}

In this appendix we provide the proofs to all propositions in the paper.

Proof. (Theorem 1) We have for $k \geq 1$ the recursion

$$
S^{(k)}=S^{(k-1)}+J^{(k)} \frac{\vartheta^{k}}{k !} .
$$

By definition, $S^{(k)}$ is up to terms of order $O\left(\vartheta^{k+1}\right)$ a solution of the $k$-th order hierarchy equation

$$
\begin{aligned}
0= & \frac{(1-\gamma)\left(S_{W}^{(k)}\right)^{\frac{\gamma}{\gamma-1}}-1}{\gamma}-\rho S^{(k)}-\frac{q\left(S_{W}^{(k)}\right)^{2}}{S_{W W}^{(k)}-\vartheta \cdot\left(S_{W}^{(k)}\right)^{2}} \\
& +r W S_{W}^{(k)},
\end{aligned}
$$

for any $k \geq 1$. We next expand (38) term by term in $\vartheta$. Using the expansion

$$
\left(S_{W}^{(k)}\right)^{\frac{\gamma}{\gamma-1}}=\left(S_{W}^{(k-1)}\right)^{\frac{\gamma}{\gamma-1}}+\frac{\gamma}{(\gamma-1)}\left(J_{W}^{(0)}\right)^{\frac{1}{\gamma-1}} J_{W}^{(k)} \frac{\vartheta^{k}}{k !}+O\left(\vartheta^{k+1}\right),
$$

this gives for instance

$$
\begin{aligned}
\frac{(1-\gamma)\left(S_{W}^{(k)}\right)^{\frac{\gamma}{\gamma-1}}-1}{\gamma}= & \frac{(1-\gamma)\left(S_{W}^{(k-1)}\right)^{\frac{\gamma}{\gamma-1}}-1}{\gamma}-\left(J_{W}^{(0)}\right)^{\frac{1}{\gamma-1}} J_{W}^{(k)} \frac{\vartheta^{k}}{k !} \\
& +O\left(\vartheta^{k+1}\right) .
\end{aligned}
$$

Doing similar expansions for all terms in (38) it follows

$$
\begin{aligned}
& O\left(\vartheta^{k+1}\right)=R^{(k-1)}(\vartheta) \\
& -\left(\rho J^{(k)}+\left(\left(J_{W}^{(0)}\right)^{\frac{1}{\gamma-1}}-r W+2 q \frac{J_{W}^{(0)}}{J_{W W}^{(0)}}\right) J_{W}^{(k)}-q \frac{\left(J_{W}^{(0)}\right)^{2}}{\left(J_{W W}^{(0)}\right)^{2}} J_{W W}^{(k)}\right) \frac{\vartheta^{k}}{k !} .
\end{aligned}
$$

Hence, $J_{W}^{(k)}$ is the solution of the inhomogeneous linear differential equation

$$
\begin{aligned}
R^{(k-1)}(\vartheta)= & \frac{\vartheta^{k}}{k !}\left\{\rho J^{(k)}+\left(\left(J_{W}^{(0)}\right)^{\frac{1}{\gamma-1}}-r W+2 q \frac{J_{W}^{(0)}}{J_{W W}^{(0)}}\right) J_{W}^{(k)}\right. \\
& \left.-q \frac{\left(J_{W}^{(0)}\right)^{2}}{\left(J_{W W}^{(0)}\right)^{2}} J_{W W}^{(k)}\right\}
\end{aligned}
$$


Remark that the homogeneous part of this equation is invariant with respect to $k$. Using the analytical expression for the zero order term $J^{(0)}$, this differential equation reads more explicitly:

$$
R^{(k-1)}(\vartheta)=\frac{\vartheta^{k}}{k !}\left(\rho J^{(k)}+a_{1} W J_{W}^{(k)}-a_{2} W^{2} J_{W W}^{(k)}\right),
$$

with $a_{1}, a_{2}$ given in Theorem 1 . This proves part 1 of the theorem. To prove 2., we expand the inhomogeneity of order 0 in $\vartheta$ to get:

$$
\begin{aligned}
R^{(0)}(\vartheta)= & \frac{(1-\gamma)\left(J_{W}^{(0)}\right)^{\frac{\gamma}{\gamma-1}}-1}{\gamma}-\rho J^{(0)}-\frac{q\left(J_{W}^{(0)}\right)^{2}}{\left(J_{W W}^{(0)}-\vartheta \cdot\left(J_{W}^{(0)}\right)^{2}\right)} \\
= & \frac{(1-\gamma)\left(J_{W}^{(0)}\right)^{\frac{\gamma}{\gamma-1}}-1}{\gamma}-\rho J^{(0)}-\frac{q\left(J_{W}^{(0)}\right)^{2}}{2 J_{W W}^{(0)}}\left(1+\vartheta \cdot \frac{\left(J_{W}^{(0)}\right)^{2}}{J_{W W}^{(0)}}\right) \\
& +O\left(\vartheta^{2}\right) \\
= & -\frac{\vartheta}{2} q\left(\frac{\left(J_{W}^{(0)}\right)^{2}}{J_{W W}^{(0)}}\right)^{2}+O\left(\vartheta^{2}\right),
\end{aligned}
$$

where we used that $J^{(0)}$ satisfies the standard Merton's differential equation. Inserting the analytical expression for $J^{(0)}$ gives

$$
R^{(0)}=-\frac{\vartheta}{2} \frac{(\alpha-r)^{2}}{\sigma^{2}} \frac{1}{(\gamma-1)^{2}}\left(\frac{\left(e^{g_{\gamma, 0}} W\right)^{\gamma}}{\rho}\right)^{2}+O\left(\vartheta^{2}\right) .
$$

This proves part 2. To show the third claim we note that the general solution $J^{(1)}$ is (using the variation of constants method)

$$
\begin{aligned}
J^{(1)} & =d_{1} W^{\frac{1-\delta_{1}-\sqrt{\left(\delta_{1}-1\right)^{2}-4 \delta_{2}}}{2}}+d_{2} W^{\frac{1-\delta_{1}+\sqrt{\left(\delta_{1}-1\right)^{2}-4 \delta_{2}}}{2}} \\
& +\frac{\left(e^{2 g_{\gamma, 0}} W^{2}\right)^{\gamma}}{\rho^{2}} \cdot \frac{1}{2 \gamma\left(2 \gamma+\delta_{1}-1\right)+\delta_{2}}
\end{aligned}
$$

for some constants $d_{1}, d_{2}$. For $\gamma \rightarrow 0$ the following limits hold

$$
\begin{aligned}
e^{2 \gamma g_{\gamma, 0}} & =\exp \left[2 \gamma\left(\ln (\rho)+\frac{(\gamma-1)}{\gamma} \ln \left(\frac{\gamma}{(1-\gamma)}\left(\frac{1}{\gamma}+\frac{q}{(\gamma-1) \rho}-\frac{r}{\rho}\right)\right)\right)\right] \rightarrow 1 \\
\delta_{1} & =-\frac{\left(\frac{e^{\gamma g_{\gamma, 0}}}{\rho}\right)^{\frac{1}{\gamma-1}}-r+\frac{2 q}{(\gamma-1)}}{\frac{(\alpha-r)^{2}}{2(\gamma-1)^{2} \sigma^{2}}} \rightarrow \delta_{1}^{*}:=-\frac{\rho-r-\frac{(\alpha-r)^{2}}{\sigma^{2}}}{q} \\
\delta_{2} & =-\frac{\rho}{\frac{(\alpha-r)^{2}}{2(\gamma-1)^{2} \sigma^{2}}} \rightarrow \delta_{2}^{*}:=-\frac{\rho}{q}
\end{aligned}
$$


implying

$$
J^{(1)} \rightarrow J^{(1)^{*}}:=d_{1} W^{\frac{1-\delta_{1}^{*}-\sqrt{\left(\delta_{1}^{*}-1\right)^{2}-4 \delta_{2}^{*}}}{2}}+d_{2} W^{\frac{1-\delta_{1}^{*}+\sqrt{\left(\delta_{1}-1\right)^{2}-4 \delta_{2}^{*}}}{2}}+\frac{1}{\rho^{2} \delta_{2}^{*}},(\gamma \rightarrow 0) .
$$

As a consequence, to satisfy the boundary condition $\lim _{\gamma \rightarrow 0} S^{(1)}=J_{l o g}^{(1)}$, we have to choose $d_{1}=d_{2}=0$. This concludes the proof of Theorem 1 .

Proof. (Proposition 3) For the optimal consumption asymptotics it follows

$$
\begin{aligned}
c & =\left(J_{W}^{(0)}+\vartheta J_{W}^{(1)}\right)^{\frac{1}{\gamma-1}}+O\left(\vartheta^{2}\right) \\
& =\left(\frac{e^{\gamma g_{\gamma, 0}}}{\rho}\right)^{\frac{1}{\gamma-1}} W\left(1-\frac{\vartheta \rho}{(1-\gamma) C_{1}}\left(e^{g_{\gamma, 0}} W\right)^{\gamma}\right)+O\left(\vartheta^{2}\right) .
\end{aligned}
$$

For the optimal allocation to risky assets we have

$$
\begin{aligned}
w & =-\frac{(\alpha-r)}{\sigma^{2}} \frac{J_{W}}{W J_{W W}\left(1-\vartheta \cdot \frac{J_{W}^{2}}{J_{W W}}\right)} \\
& =-\frac{(\alpha-r)}{\sigma^{2} W} \frac{J_{W}^{(0)}}{J_{W W}^{(0)}}\left(1+\vartheta\left(\frac{\left(J_{W}^{(0)}\right)^{2}}{J_{W W}^{(0)}}-\frac{J_{W W}^{(1)}}{J_{W W}^{(0)}}+\frac{J_{W}^{(1)}}{J_{W}^{(0)}}\right)\right)+O\left(\vartheta^{2}\right) .
\end{aligned}
$$

The analytical expressions for $J^{(0)}, J^{(1)}$ then imply

$$
w=\frac{(\alpha-r)}{\sigma^{2}(1-\gamma)}\left(1+\vartheta \cdot \frac{C_{1}-2 \gamma^{2} \rho}{\rho(\gamma-1) C_{1}}\left(e^{g_{\gamma, 0}} W\right)^{\gamma}\right)+O\left(\vartheta^{2}\right) .
$$

Proof. (Lemma 4) By definition $R^{(k)}$ is given by

$$
R^{(k)}=\underbrace{\frac{(1-\gamma)\left(S_{W}^{(k)}\right)^{\frac{\gamma}{\gamma-1}}-1}{\gamma}}_{A^{(k)}} \underbrace{-\rho S^{(k)}+r W S_{W}^{(k)}}_{B^{(k)}}-q \underbrace{\frac{\left(S_{W}^{(k)}\right)^{2}}{\left(S_{W W}^{(k)}-\vartheta \cdot\left(S_{W}^{(k)}\right)^{2}\right)}}_{C^{(k)}} .
$$

We expand $R^{(k)}$ term by term up to order $O\left(\vartheta^{k+2}\right)$. For any $k \geq 1$ it then 
follows using the definition of $S^{(k)}$

$$
\begin{aligned}
A^{(k)}= & \frac{(1-\gamma)\left(S_{W}^{(k)}\right)^{\frac{\gamma}{\gamma-1}}-1}{\gamma} \\
= & \frac{(1-\gamma)\left(S_{W}^{(k-1)}\right)^{\frac{\gamma}{\gamma-1}}-1}{\gamma}-\left(S_{W}^{(k-1)}\right)^{\frac{1}{\gamma-1}} J_{W}^{(k)} \frac{\vartheta^{k}}{k !} \\
& -\frac{1}{2} \frac{1}{\gamma-1}\left(S_{W}^{(k-1)}\right)^{\frac{1}{\gamma-1}-1}\left(J_{W}^{(k)} \frac{\vartheta^{k}}{k !}\right)^{2}+O\left(\vartheta^{2 k+1}\right) \\
= & A^{(k-1)}-\frac{\vartheta^{k}}{k !}\left(S_{W}^{(1)}\right)^{\frac{1}{\gamma-1}} J_{W}^{(k)}+O\left(\vartheta^{2 k}\right) \\
= & A^{(k-1)}-\frac{\vartheta^{k}}{k !}\left(J_{W}^{(0)}\right)^{\frac{1}{\gamma-1}} J_{W}^{(k)}-\frac{\vartheta^{k+1}}{k !} \frac{1}{\gamma-1}\left(J_{W}^{(0)}\right)^{\frac{1}{\gamma-1}-1} J_{W}^{(1)} J_{W}^{(k)} \\
& +O\left(\vartheta^{k+2}\right) \\
= & A^{(k-1)}-\frac{\vartheta^{k}}{k !}\left(J_{W}^{(0)}\right)^{\frac{1}{\gamma-1}} J_{W}^{(k)}-\frac{\vartheta^{k+1}}{k !} A_{1} J_{W}^{(k)}+O\left(\vartheta^{k+2}\right),
\end{aligned}
$$

where $A_{1}$ is a function of $J^{(0)}$ and $J^{(1)}$ only. For $B^{(k)}$ we have

$$
B^{(k)}=-\rho S^{(k)}+r W S_{W}^{(k)}=B^{(k-1)}+\frac{\vartheta^{k}}{k !}\left(-\rho J^{(k)}+r W J_{W}^{(k)}\right) .
$$

Finally, after some computations

$$
\begin{aligned}
C^{(k)}= & \frac{\left(S_{W}^{(k)}\right)^{2}}{\left(S_{W W}^{(k)}-\vartheta \cdot\left(S_{W}^{(k)}\right)^{2}\right)} \\
= & \frac{\left(S_{W}^{(k-1)}\right)^{2}+2 S_{W}^{(k-1)} \frac{\vartheta^{k}}{k !} J_{W}^{(k)}}{S_{W W}^{(k-1)}+\frac{\vartheta^{k}}{k !} J_{W W}^{(k)}-\vartheta \cdot\left(S_{W}^{(k-1)}\right)^{2}-2 S_{W}^{(k-1)} \frac{\vartheta^{k+1}}{k !} J_{W}^{(k)}}+O\left(\vartheta^{2 k+1}\right) \\
= & C^{(k-1)} \\
& +\frac{2 S_{W}^{(1)} \frac{\vartheta^{k}}{k !} J_{W}^{(k)}}{S_{W W}^{(1)}-\vartheta \cdot\left(J_{W}^{(0)}\right)^{2}}-\frac{\vartheta^{k}}{k !} \frac{\left(S_{W}^{(1)}\right)^{2}\left(J_{W W}^{(k)}-2 \vartheta J_{W}^{(0)} J_{W}^{(k)}\right)}{\left(J_{W W}^{(0)}\right)^{2}\left(1+2 \vartheta\left(\frac{J_{W W}^{(1)}}{J_{W W}^{(0)}}-\frac{\left(J_{W}^{(0)}\right)^{2}}{J_{W W}^{(0)}}\right)\right)} \\
& +O\left(\vartheta^{k+2}\right) \\
= & C^{(k-1)}+2 D^{(k)}-E^{(k)}+O\left(\vartheta^{k+2}\right) .
\end{aligned}
$$

We now further expand the RHS of the last equality for $C^{(k)}$ term by term. It 
first follows

$$
\begin{aligned}
D^{(k)} & =\frac{S_{W}^{(1)} \frac{\vartheta^{k}}{k !} J_{W}^{(k)}}{S_{W W}^{(1)}-\vartheta \cdot\left(J_{W}^{(0)}\right)^{2}} \\
& =\frac{J_{W}^{(0)} \frac{\vartheta^{k}}{k !} J_{W}^{(k)}+J_{W}^{(1)} \frac{\vartheta^{k+1}}{k !} J_{W}^{(k)}}{J_{W W}^{(0)}-\vartheta \cdot\left(\left(J_{W}^{(0)}\right)^{2}-J_{W W}^{(1)}\right)} \\
& =\frac{J_{W}^{(0)}}{J_{W W}^{(0)}} \frac{\vartheta^{k}}{k !} J_{W}^{(k)}+D_{1} \frac{\vartheta^{k+1}}{k !} J_{W}^{(k)}+O\left(\vartheta^{k+2}\right)
\end{aligned}
$$

with $D_{1}$ defined accordingly. We also have for the remaining term

$$
\begin{aligned}
E^{(k)} & =\frac{\vartheta^{k}}{k !} \frac{\left(J_{W W}^{(k)}-2 \vartheta J_{W}^{(0)} J_{W}^{(k)}\right)\left(S_{W}^{(1)}\right)^{2}}{\left(J_{W W}^{(0)}\right)^{2}\left(1+2 \vartheta\left(\frac{J_{W W}^{(1)}}{J_{W W}^{(0)}}-\frac{\left(J_{W}^{(0)}\right)^{2}}{J_{W W}^{(0)}}\right)\right)} \\
& =\frac{\vartheta^{k}}{k !} \frac{\left(J_{W}^{(0)}\right)^{2}}{\left(J_{W W}^{(0)}\right)^{2}} J_{W W}^{(k)}-\frac{\vartheta^{k+1}}{k !}\left(E_{1} J_{W}^{(k)}-E_{2} J_{W W}^{(k)}\right)+O\left(\vartheta^{k+2}\right),
\end{aligned}
$$

with $E_{1}, E_{2}$ defined accordingly. Thus, by collecting terms we obtain

$$
\begin{aligned}
R^{(k)}= & R^{(k-1)} \\
& -\frac{\vartheta^{k}}{k !}\left(\rho J^{(k)}+\left(\left(J_{W}^{(0)}\right)^{\frac{1}{\gamma-1}}-r W+2 q \frac{J_{W}^{(0)}}{J_{W W}^{(0)}}\right) J_{W}^{(k)}-q \frac{\left(J_{W}^{(0)}\right)^{2}}{\left(J_{W W}^{(0)}\right)^{2}} J_{W W}^{(k)}\right) \\
& -\frac{\vartheta^{k+1}}{k !}\left(\left(A_{1}+2 q D_{1}+E_{1}\right) J_{W}^{(k)}-E_{2} J_{W W}^{(k)}\right) \\
& +O\left(\vartheta^{k+2}\right) \\
= & -\frac{\vartheta^{k+1}}{k !}\left(\left(A_{1}+2 q D_{1}+E_{1}\right) J_{W}^{(k)}-E_{2} J_{W W}^{(k)}\right)+O\left(\vartheta^{k+2}\right)
\end{aligned}
$$

using again Theorem 1 . Computing $A_{1}, D_{1}, E_{1}, E_{2}$, explicitly it follows from 
the explicit form for $J^{(1)}$ in Theorem 1 ,

$$
\begin{aligned}
& A_{1}=\frac{1}{\gamma-1}\left(J_{W}^{(0)}\right)^{\frac{1}{\gamma-1}} \frac{J_{W}^{(1)}}{J_{W}^{(0)}}=\frac{2 \gamma \rho}{(\gamma-1) C_{1}}\left(\frac{1}{\rho} e^{\gamma g_{\gamma, 0}}\right)^{\frac{\gamma}{\gamma-1}} e^{\gamma g_{\gamma, 0}} W^{\gamma+1} \\
& D_{1}=\frac{J_{W}^{(1)}}{J_{W W}^{(0)}}+\frac{\left(\left(J_{W}^{(0)}\right)^{2}-J_{W W}^{(1)}\right) J_{W}^{(0)}}{\left(J_{W W}^{(0)}\right)^{2}}=\frac{\rho e^{\gamma g_{\gamma, 0}}}{(\gamma-1)^{2}}\left(\frac{1}{\rho^{2}}-\frac{2 \gamma^{2}}{C_{1}}\right) W^{\gamma+1} \\
& E_{1}=2 \frac{\left(J_{W}^{(0)}\right)^{3}}{\left(J_{W W}^{(0)}\right)^{2}}=2 \frac{\left(\frac{1}{\rho} e^{\gamma g_{\gamma, 0}}\right)^{3} W^{3 \gamma-3}}{\left(\frac{\gamma-1}{\rho} e^{\gamma g_{\gamma, 0}}\right)^{2} W^{2 \gamma-4}}=\frac{1}{\rho(\gamma-1)^{2}} e^{\gamma g_{\gamma, 0}} W^{\gamma+1}
\end{aligned}
$$

and

$$
\begin{aligned}
E_{2} & =2\left(\frac{J_{W}^{(0)} J_{W}^{(1)}}{\left(J_{W W}^{(0)}\right)^{2}}-\frac{J_{W W}^{(1)}-\left(J_{W}^{(0)}\right)^{2}}{\left(J_{W W}^{(0)}\right)^{3}}\right) \\
& =\frac{2 \rho e^{\gamma g_{\gamma, 0}}}{(\gamma-1)^{2} C_{1}} W^{\gamma+2}-\left(\frac{\rho}{\gamma-1}\right)^{3} \frac{2}{e^{\gamma g_{\gamma, 0}}}\left(\frac{2 \gamma(2 \gamma-1)}{C_{1}}-\frac{1}{\rho^{2}}\right) W^{-(\gamma-4)}
\end{aligned}
$$

Summarizing, we thus obtain

$$
R^{k}=-\frac{\vartheta^{k+1}}{k !} \mathbf{M}\left(J^{(k)}\right)+O\left(\vartheta^{k+2}\right),
$$

where $\mathbf{M}$ is the linear differential operator defined by

$$
\mathbf{M}(J)=\left(A_{1}+q\left(2 D_{1}+E_{1}\right)\right) W^{\gamma+1} J_{W}-q\left(F_{1} W^{\gamma+2}-F_{2} W^{-(\gamma-4)}\right) J_{W W},
$$

where the constants $A_{1}, D_{1}, E_{1}$, are given explicitly above and

$$
F_{1}=\frac{2 \rho e^{\gamma g_{\gamma, 0}}}{(\gamma-1)^{2} C_{1}} \quad, \quad F_{2}=\left(\frac{\rho}{\gamma-1}\right)^{3} \frac{2}{e^{\gamma g_{\gamma, 0}}}\left(\frac{2 \gamma(2 \gamma-1)}{C_{1}}-\frac{1}{\rho^{2}}\right)
$$

Proof. (Lemma 5) We first note that the action of the operator $\mathbf{M}$ on $W^{\mu}$ for $\mu \in \mathbf{R}$ is of the form

$$
\mathbf{M}\left(W^{\mu}\right)=e_{i_{1}} W^{\gamma+\mu}+e_{i_{2}} W^{\gamma+\mu-6},
$$

with $e_{i_{1}}, e_{i_{2}}$ defined in Lemma 5 . The proof of the Lemma is then obtained by induction starting from a 'correct guess' for (29). Precisely, start by computing $\mathbf{M}\left(J^{(0)}\right)$ in (28) using (39). Then, compute in the next step

$$
W^{a_{1}} \int W^{b_{1}} \mathbf{M}\left(J^{(0)}\right) d W
$$


and so on. By applying recursively this induction step to (28) the expression (29) is then obtained.

Proof. (Corollary 6) The exponent of $W$ in (29) is positive after two differentiations if and only if

$$
(k+1)\left(1-\delta_{1}\right)+2 \gamma-6+k(\gamma-6)+k-3>0,
$$

where we used the expressions for $a, b$ given in Theorem 1 and the fact that $m_{i_{n}}=m_{2}=\gamma-6$. Rearranging terms and noting that $a_{2}>0$ we get

$$
\frac{a_{1}}{a_{2}}>\frac{1}{k+1}(9-2 \gamma)-1-\frac{k}{k+1}(\gamma-5) \text {. }
$$

Since the right hand side is monotone in $k$, the proof follows by setting $k=1$.

Proof. (Theorem 7) We first consider the case $W>1$. The assumptions guarantee that there are no singularities for any $J^{(k)}, k \geq 1$, and Lemma 5 implies:

$$
\begin{aligned}
& |J(W)| \\
& \leq e^{\gamma g_{\gamma}, 0} \sum_{k=0}^{\infty} \sum_{\left(a_{k}, b_{k}\right) \in A} \sum_{\left(a_{k-1}, b_{k-1}\right) \in A} \ldots \sum_{\left(a_{1}, b_{1}\right) \in A} \sum_{i_{k}=1}^{2} \sum_{i_{k-1}=1}^{2} \ldots \sum_{i_{1}=1}^{2} \\
& \times \frac{\vartheta^{k}}{k !}\left|\frac{e_{i_{1}} W^{h_{i_{1}}^{0}+\sum_{n=0}^{k}\left(a_{n}+b_{n}\right)+\sum_{n=0}^{k-1} m_{i_{n}}+k-1}}{\prod_{m=0}^{k}\left(h_{i_{1}}^{0}+\sum_{n=0}^{m-1} b_{n}+\sum_{n=0}^{m-1} m_{i_{n}}+m\right)}\right| \\
& \leq e^{\gamma g_{\gamma, 0}} \sum_{k=0}^{\infty} 2^{2 k} \frac{\vartheta^{k}}{k !} \\
& \times \max _{a_{j}, b_{j}, i_{j}: j=1, \ldots, k}\left|\frac{e_{i_{1}} W^{h_{i_{1}}^{0}+\sum_{n=0}^{k}\left(a_{n}+b_{n}\right)+\sum_{n=0}^{k-1} m_{i_{n}}+k-1}}{\prod_{m=0}^{k}\left(h_{i_{1}}^{0}+\sum_{n=0}^{m-1} b_{n}+\sum_{n=0}^{m-1} m_{i_{n}}+m\right)}\right|,
\end{aligned}
$$

where we used that there are $2^{2 k}$ terms hidden in all summations apart for the summation over $k$. Inserting the terms which lead to the maximum it follows

$$
\begin{aligned}
|J(W)| & \leq e^{\gamma g_{\gamma}, 0} \sum_{k=0}^{\infty} 2^{2 k} \frac{\vartheta^{k}}{k !}\left|\frac{e_{1_{1}}+e_{1_{2}}}{(3 \gamma+b+k)^{k}} W^{2 \gamma+(k+1)(a+b)+k \gamma}\right| \\
& \leq e^{\gamma g_{\gamma, 0}}\left|e_{1_{1}}+e_{1_{2}}\right| \sum_{k=0}^{\infty} 2^{2 k} \frac{\vartheta^{k}}{k !} \frac{1}{(3 \gamma+b)^{k}} W^{2 \gamma+(k+1)(a+b)+k \gamma} \\
& \leq e^{\gamma g_{\gamma, 0}}\left|e_{1_{1}}+e_{1_{2}}\right| W^{2 \gamma+a+b} e^{4 \vartheta \frac{1}{3 \gamma+b} W^{a+b+\gamma}}<\infty
\end{aligned}
$$


Therefore, the perturbation series converges for $W>1$ under the given conditions. For $0<W \leq 1$ a similar bound can be derived, to get:

$$
\begin{aligned}
& \leq \quad|J(W)| \\
& \leq \quad e^{\gamma g_{\gamma, 0}} \sum_{k=0}^{\infty} 2^{2 k} \frac{\vartheta^{k}}{k !} \\
& \times \quad \max _{a_{j}, b_{j}, i_{j}: j=1, \ldots, k}\left|\frac{e_{i_{1}} W^{h_{i_{1}}^{0}+\sum_{n=0}^{k}\left(a_{n}+b_{n}\right)+\sum_{n=0}^{k-1} m_{i_{n}+k-1}}}{\prod_{m=0}^{k}\left(h_{i_{1}}^{0}+\sum_{n=0}^{m-1} b_{n}+\sum_{n=0}^{m-1} m_{i_{n}}+m\right)}\right| \\
& \leq \quad e^{\gamma g_{\gamma, 0}} \sum_{k=0}^{\infty} 2^{2 k} \frac{\vartheta^{k}}{k !}\left|\frac{e_{1_{1}}+e_{1_{2}}}{(3 \gamma+b-12)^{k}} W^{2 \gamma-6+(k+1)(a+b)+k(\gamma-6)}\right| \\
& \leq \quad e^{\gamma g_{\gamma, 0}}\left|e_{1_{1}}+e_{1_{2}}\right| W^{2 \gamma-6+a+b} e^{4 \vartheta \frac{1}{3 \gamma+b-12} W^{a+b+\gamma-6}}<\infty .
\end{aligned}
$$

The claims of the theorem follows from the $W$-exponent in Lemma 5 and by noting that under the given conditions the power series of the first two derivatives of $J^{(k)}, k \geq 1$, can be bounded in a similar way. 


\section{References}

[1] Anderson, E., L.P. Hansen and T. Sargent, (1998). Risk and robustness in geneal equilibrium, mimeo, University of Chicago.

[2] Anderson, E., L.P. Hansen and T. Sargent, (2000). Robustness, detection and the price of risk, mimeo, University of Chicago.

[3] Chen, Z. and L. G. Epstein, (2000). Ambiguity, risk and asset returns in continuous time, mimeo, University of Rochester.

[4] Cox, J. C. and C. Huang, (1989). Optional consumption and portfolio policies when assets prices follow a diffusion process, Journal of Economic Theory, 49, 33-83.

[5] Cuoco, D., (1997). Optimal consumption and equilibrium prices with portfolio constraints and stochastic income, Journal of Economic Theory, 72, 33-73.

[6] Duffie, D. and L. G. Epstein, (1992). Stochastic differential utility, Econometrica 60, 353-394.

[7] Fleming, W.H. and P.E. Souganidis, (1989). On the existence of value functions of two-player, zero-sum stochastic differential games, Indiana Univ. Math. Journal, 38, 293-314.

[8] Fleming, W.H. and H.M. Soner, (1992). Controlled Markov processes and viscosity solutions, Springer-Verlag, New York.

[9] Gilboa, I. and D. Schmeidler, (1989). Maxmin expected utility with nonunique prior, Journal of Mathematical Economics, 18, 141-153.

[10] Hansen, L. P., T. J. Sargent, and D. Tallarini, Jr., (1999) Robust Permanent Income and Pricing, Review of Economic Studies, 66, 873-907.

[11] Hansen, L. P., T.J. Sargent, G. A. Turmuhambetova and N. Williams, (2001). Robustness and uncertainty aversion, mimeo University of Chicago.

[12] Kim, T. S. and E. Omberg, (1996). Dynamic nonmyopic portfolio behavior, Review of Financial Studies 9, 141-161.

[13] Kogan and Uppal (2000). Risk aversion and optimal portfolio policies in partial and general equilibrium economies, Preprint, University of British Columbia, Vancouver.

[14] Liu, J., (1998). Portfolio selection in stochastic environments, Working paper, Stanford University.

[15] Merton, R., (1969) . Lifetime portfolio selection under uncertainty: the continuous time case, Review of Economics and Statistics, 51, 247-257. 
[16] Merton, R. C., (1971). Optimum consumption and portfolio rules in a continuous time model, Journal of Economic Theory 3, 373-413.

[17] Schroder, M. and C. Skiadas (1999) Optimal Consumption and Portfolio Selection with Stochastic Differential Utility, Journal of Economic Theory, 89 , No. $1,68-126$.

[18] Souganidis, P.E., (1999). Two-player, zero-sum differential games and viscosity solutions, in Stochastic Differential Games: Theory and Numerical Methods, Bardi, M. et al. (editors), Annals of the International Society of Dynamic Games, Birkhäuser, Boston.

[19] Wachter, J., (1998). Portfolio and consumption decisions under meanreverting returns: An exact solution for complete markets, Working paper, Harvard University.

[20] Yong, J. and X.Y. Zhou, (1999). Stochastic controls: Hamilton systems and HJB equations, Applications of Mathematics, Stochastic Modelling and Applied Probability, vol. 43, Springer, New York. 